\title{
LEAD CARBONATE, A NEW FAST, HEAVY SCINTILLATOR
}

\author{
W.W. Moses and S.E. Derenzo, \\ Research Medicine and Radiation Biology Division, \\ Lawrence Berkeley Laboratory, \\ 1 Cyclotron Road, Berkeley, CA 94720
}

\begin{abstract}
We describe the scintillation properties of lead carbonate $\left(\mathrm{PbCO}_{3}\right)$, a newly discovered, heavy $\left(6.6 \mathrm{~g} / \mathrm{cm}^{3}\right)$, inorganic scintillator. Its fluorescence decay lifetime, measured with the delayed coincidence method, is described by a sum of three exponentials; $24 \%$ of the light is emitted with a $5.6 \pm 1$ ns time constant, $48 \%$ of the light is emitted with a $27 \pm 2 \mathrm{~ns}$ time constant, and $28 \%$ is emitted with a $155 \pm 10$ ns time constant. The emission spectrum peak is centered at a wavelength of $475 \mathrm{~nm}$, and drops to less than $10 \%$ of its peak value at $370 \mathrm{~nm}$ and $580 \mathrm{~nm}$. We have been unable to obtain an optical quality crystal of pure $\mathrm{PbCO}_{3}$, but when a $3 \mathrm{~mm}$ cube of a cerussite (a naturally occurring mineral form of $\mathrm{PbCO}_{3}$ ) is excited with $511 \mathrm{keV}$ photons, a photopeak with a $42 \%$ full width at half maximum is observed at approximately $9 \%$ the light output of a bismuth germanate (BGO) crystal with similar geometry. The light output increases rapidly with decreasing temperature, plateauing at twice the light output of BGO at approximately $-40^{\circ} \mathrm{C}$. Lead hydroxide $\left(\mathrm{Pb}(\mathrm{OH})_{2}\right)$ can be mixed with $\mathrm{PbCO}_{3}$ in a $1: 2$ ratio without significantly affecting the scintillation properties. The short fluorescence lifetime, high density, and reasonable light output of this new scintillator suggest that it would be useful for applications where high counting rates, good stopping power, and nanosecond timing are important, such as medical imaging and nuclear science.
\end{abstract}

\section{Introduction}

This paper describes the scintillation properties of lead carbonate $\left(\mathrm{PbCO}_{3}\right)$, a newly discovered inorganic scintillator. The physical characteristics of $\mathrm{PbCO}_{3}$ are well suited for use as a gamma radiation detector. It has a density of $6.6 \mathrm{~g} / \mathrm{cm}^{3}$, is not hygroscopic, and is birefringent with indices of refraction of 1.80 and 2.08 [1]. The crystal structure is rhombic [1], its attenuation length for $511 \mathrm{keV}$ photons is $1.1 \mathrm{~cm}$, and it is colorless, transmitting wavelengths

*This work was supported in part by the U.S. Department of Energy, under contracts No. DE-AC03-76SF00098 and DE-ACO382ER-13000, and in part by Public Health Service Grant Nos. P01 25840 and R01 CA38086.

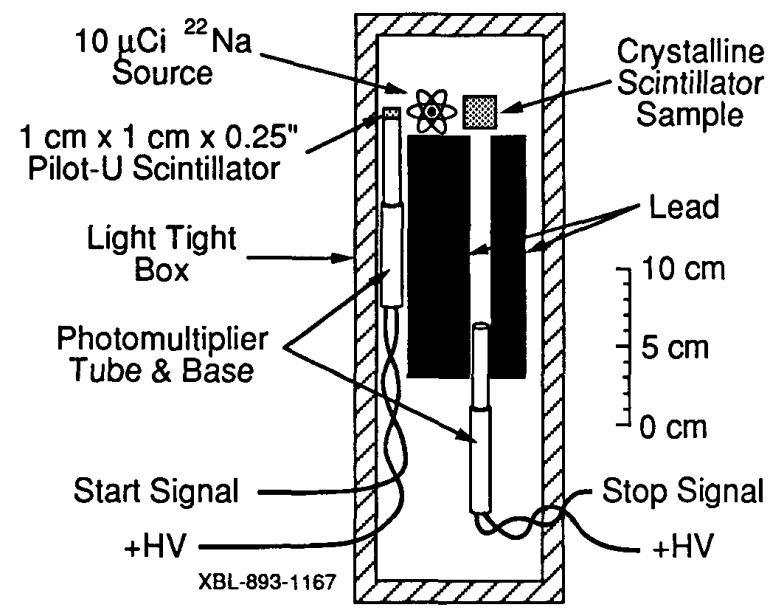

Figure 1: Delayed-Coincidence Apparatus

down to approximately $270 \mathrm{~nm}$. We have been unable to obtain an optical quality synthetic crystal of pure lead carbonate, and therefore have performed our measurements using crystals cut from a naturally occurring mineral form of $\mathrm{PbCO}_{3}$ known as cerussite. Also, all measurements were performed at room temperature $\left(24^{\circ} \mathrm{C}\right)$ unless otherwise indicated.

\section{Fluorescent Decay Time}

The fluorescent decay lifetime was measured using the delayed-coincidence method of Bollinger and Thomas [2], as modified by Moszyński and Bengtson [3]. A diagram of this set-up is shown in Figure 1. A piece of Pilot-U plastic scintillator coupled to a Hamamatsu R-2055 photomultiplier tube provided a start signal, and another quartzwindowed Hamamatsu R-2055 photomultiplier tube placed $13 \mathrm{~cm}$ away from the $\mathrm{PbCO}_{3}$ sample provided the stop signal. A $10 \mu \mathrm{Ci}^{22} \mathrm{Na}$ source provided the $511 \mathrm{keV}$ photon pairs that excited both the plastic scintillator and the scintillator sample. Timing signals from both photomultiplier tubes were generated using two channels of a Tennelec TC454 constant fraction discriminator, and the time difference 


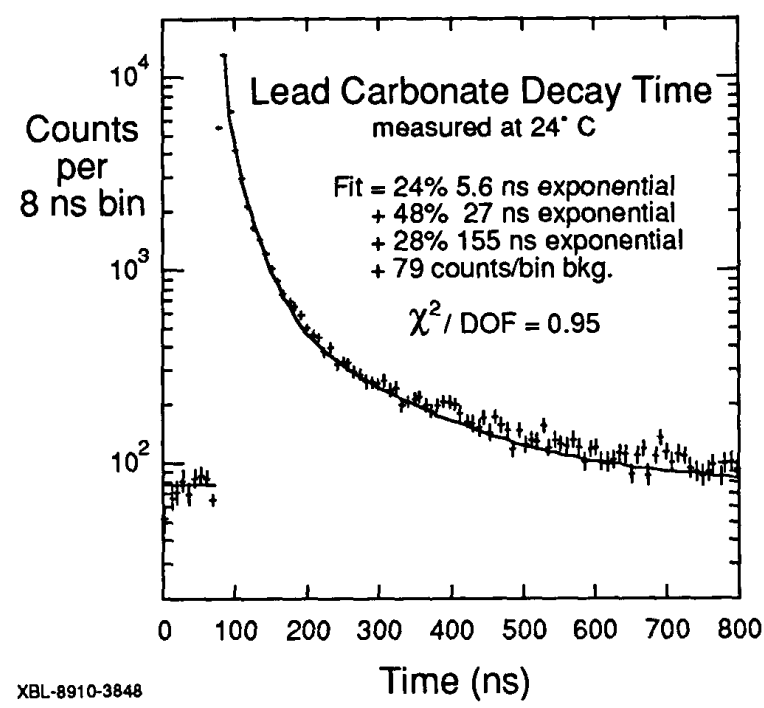

Figure 2: Fluorescence Decay Time of $\mathrm{PbCO}_{3}$

between the start and stop signals was digitized with an Ortec 457 time to amplitude converter and a LeCroy 3512 analog to digital converter (ADC).

The results of this measurement are shown in Figure 2. A good fit to the data (the chi-squared per degree of freedom is 0.95 ) is obtained with a sum of three exponential decay lifetimes plus a constant background. Note that although the data Figure 2 is displayed in $8 \mathrm{~ns}$ bins, the data was acquired and fit using 0.4 ns bins. A significant fraction of the light ( $24 \%$ of the emitted photons) is produced with an $5.6 \pm 1.0$ ns decay constant, while $48 \%$ of the emitted photons are produced with a $27 \pm 2$ ns decay constant and $28 \%$ are produced with a $155 \pm 10$ ns time constant. The errors in this measurement are dominated by correlations between the three exponential terms.

\section{Coincidence Timing}

The coincidence resolving time of $\mathrm{PbCO}_{3}$ was measured by exciting two crystals of $\mathrm{PbCO}_{3}$, each coupled to a quartz windowed Hamamatsu R-2059 photomultiplier tube operated at $-2500 \mathrm{~V}$, with $511 \mathrm{keV}$ photons resulting from positron annihilation from a ${ }^{22} \mathrm{Na}$ source placed between the two crystals. A timing signal from each photomultiplier tube was generated using two channels of a Tennelec TC-454 constant fraction discriminator, and the time difference between the two timing signals was digitized with an Ortec 457 time to amplitude converter and a LeCroy 3512 ADC. The resulting timing distribution, which has a FWHM of $1.3 \mathrm{~ns}$ and a full width at tenth maximum (FWTM) of $3.2 \mathrm{~ns}$, is plotted in Figure 3. The same apparatus measures a timing distribution FWHM of $0.5 \mathrm{~ns}$ for Barium Fluoride $\left(\mathrm{BaF}_{2}\right)$.

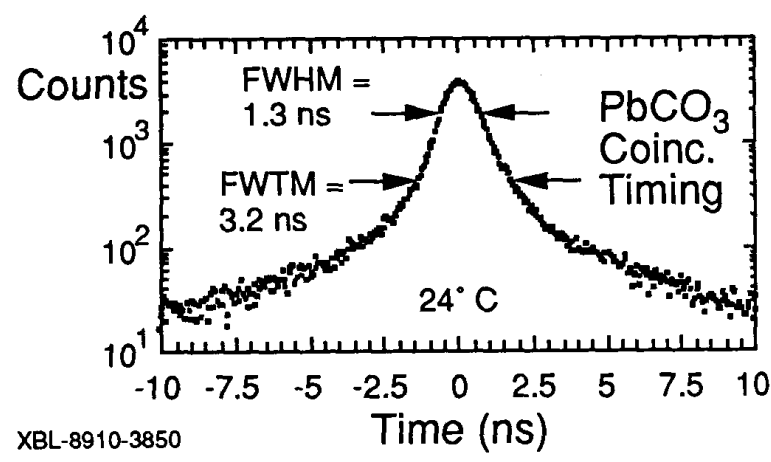

Figure 3: Coincidence Time Resolution

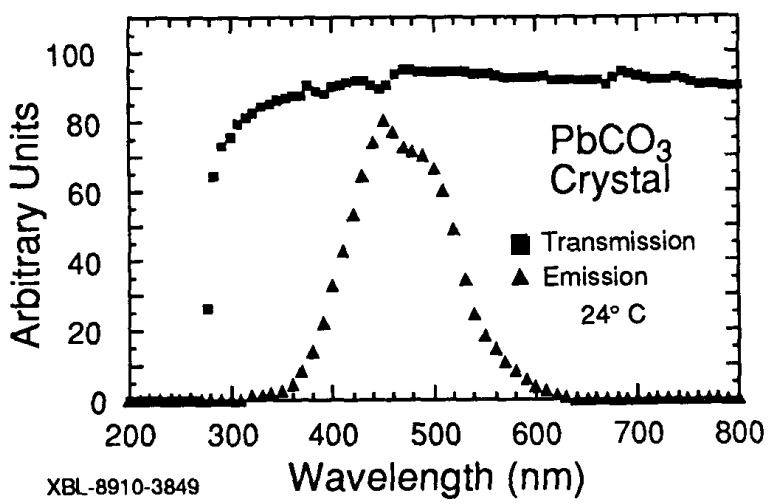

Figure 4: Emission and Transmission Spectra of $\mathrm{PbCO}_{3}$

\section{Emission Spectrum}

The emission spectrum of $\mathrm{PbCO}_{3}$ was obtained using a 0.125 meter Jarrell-Ash MonoSpec 18 monochromator with a 1200 line $/ \mathrm{mm}$ grating blazed for $500 \mathrm{~nm}$. The entrance and exit slits of this monochromator were $500 \mu \mathrm{m}$ wide, resulting in a spectral resolution of $12 \mathrm{~nm}$. The $511 \mathrm{keV}$ photons from a $3.0 \mathrm{mCi}{ }^{68} \mathrm{Ge}$ source were used to excite a small (5 mm typical dimension) crystal that was cut from a cerussite crystal. The faces of this crystal were polished and covered on 5 sides with a reflective coating of white Teflon tape. The sixth side was placed at the entrance slit of the monochromator, and a quartz windowed Hamamatsu R-2055 photomultiplier tube (spectral range $200 \mathrm{~nm}$ to $600 \mathrm{~nm}$ ) was placed at the exit slit. The resulting photomultiplier count rate is plotted, after background subtraction, as a function of monochromator wavelength in Figure 4. This spectrum is not corrected for the spectral response of the photomultiplier tube or monochromator. The emission spectrum peak is centered at $475 \mathrm{~nm}$, and drops to $10 \%$ of its maximum intensity at $370 \mathrm{~nm}$ and $580 \mathrm{~nm}$. Note that absence of light below the $300 \mathrm{~nm}$ cutoff of borosilicate glass implies that fused silica or UV glass windowed photomultiplier tubes are not necessary in order to collect all of the $\mathrm{PbCO}_{3}$ scintillation light. The emis- 
sion spectrum obtained with optical $(254 \mathrm{~nm})$ excitation is virtually identical to that obtained with $511 \mathrm{keV}$ photon excitation.

Figure 4 also shows the transmission spectrum of a $3 \mathrm{~mm}$ thick sample of $\mathrm{PbCO}_{3}$, as measured with a Shimadzu Spectronic 200UV spectrophotometer. The transmission is relatively uniform for wavelengths greater than $400 \mathrm{~nm}$, with a gradual decrease between $400 \mathrm{~nm}$ and the ultimate cutoff of $270 \mathrm{~nm}$, demonstrating that $\mathrm{PbCO}_{3}$ is transparent to its own emissions. A small amount of structure is visible at $375 \mathrm{~nm}$ and near $450 \mathrm{~nm}$ - these structures are observed in several samples, but it is not clear what they are due to. The samples have been chemically analyzed and are almost entirely $\mathrm{PbCO}_{3}$, but small amounts $(<1 \%)$ of impurities may be present.

\section{Light Output}

The light output of $\mathrm{PbCO}_{3}$ was measured by comparing its response to $511 \mathrm{keV}$ photons to the response of a bismuth germanate (BGO) crystal under the same conditions. A $3 \mathrm{~mm}$ cube of cerussite was coated on five sides with a reflective coating of white Teflon tape, then optically coupled to a quartz-windowed Hamamatsu R-1306 photomultiplier tube with General Electric Viscasil $600 \mathrm{M}$ silicone fluid. The crystal was irradiated with $511 \mathrm{keV}$ positron annihilation photons from a ${ }^{22} \mathrm{Na}$ source, and the output of the photomultiplier tube amplified with a Tennelec TC222 amplifier with $1.2 \mu \mathrm{sec}$ shaping time and digitized with a Lecroy 3512 ADC. The resulting pulse height spectrum is plotted (after pedestal subtraction) in Figure 5(a). The $\mathrm{PbCO}_{3}$ crystal was removed and the same experiment was performed on a $3 \mathrm{~mm}$ cube of $\mathrm{BGO}$, and the resulting spectrum is shown in Figure 5(b).

The photopeak corresponding to the $511 \mathrm{keV}$ photon is seen in each plot in Figure 5 . Note that the units used for the horizontal scale are the same for both plots. The $511 \mathrm{keV}$ photopeak in $\mathrm{PbCO}_{3}$ is centered at a pulse height that is $9 \%$ of the $511 \mathrm{keV}$ photopeak pulse height in BGO. Using the BGO light output of 8200 photons/MeV reported by Holl, et al. [4], this implies that the light output of $\mathrm{PbCO}_{3}$ is approximately 760 photons $/ \mathrm{MeV}$. The full width at half maximum (FWHM) in $\mathrm{PbCO}_{3}$ of the $511 \mathrm{keV}$ photopeak is $42 \%$, which is consistent with a light output that is $9 \%$ of $\mathrm{BGO}$.

The light output of $\mathrm{PbCO}_{3}$ increases significantly when it is cooled. Figure 6 compares the light output of $\mathrm{PbCO}_{3}$ at several temperatures to the light output of $\mathrm{BGO}$ at room temperature $\left(24^{\circ} \mathrm{C}\right)$. The $\mathrm{PbCO}_{3}$ light output at room temperature is $9 \%$ of $\mathrm{BGO}$, exponentially increasing to twice that of $\mathrm{BGO}$ at approximately $-40^{\circ} \mathrm{C}$, and stabilizing at temperatures below $-40^{\circ} \mathrm{C}$. The ${ }^{22} \mathrm{Na}$ excited pulse height spectrum of cooled $\mathrm{PbCO}_{3}$ is shown in Figure 5(c). Although we have not measured the effect of temperature on decay time, observation of oscilloscope traces indicates that the fast component of the decay time


Figure 5: Light Output of (a) $\mathrm{PbCO}_{3}$, (b) BGO, (c) cooled $\mathrm{PbCO}_{3}$

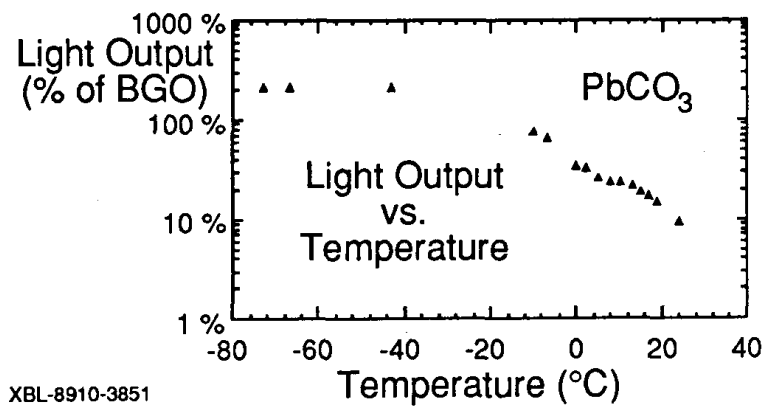

Figure 6: Light Output of $\mathrm{PbCO}_{3}$ vs. Temperature 
is not significantly changed as the temperature is reduced, but an additional slow $(\sim 1 \mu \mathrm{s})$ component is responsible for the increased light output.

\section{Basic Lead Carbonate}

Two molecules of lead carbonate can combine with a single molecule of lead hydroxide $\left(\mathrm{Pb}(\mathrm{OH})_{2}\right)$, to form a material known as basic lead carbonate, which has a density that is slightly less than pure $\mathrm{PbCO}_{3}\left(6.2 \mathrm{~g} / \mathrm{cm}^{3}\right)$. We have measured the scintillation properties of a powdered sample of pure basic lead carbonate, and found them to be very similar to those of cerussite crystals (i.e. pure $\mathrm{PbCO}_{3}$ ). The fluorescence decay time of a powdered sample of basic lead carbonate was measured using the delayed coincidence method [2] on a sample excited with a 1 ns burst of $21.7 \mathrm{keV}$ synchrotron $\mathrm{x}$-rays from beamline X23-A2 at Brookhaven National Laboratory. The resulting spectrum is very similar to the distribution shown in Figure 2, and when fit with three exponentials as in Section 2, shows $32 \%$ of the photons emitted with an $6.6 \mathrm{~ns}$ decay time, $46 \%$ emitted with a 32 ns decay time, and $22 \%$ produced with a $138 \mathrm{~ns}$ time constant $\left(\chi^{2} / \mathrm{DOF}=1.1\right)$. The emission spectrum of the powdered sample was obtained by exciting the sample with synchrotron $x$-rays, then measuring the spectral output with the apparatus used to measure the emission spectrum of the cerussite crystal in Section 4 . The resulting spectrum peaks at $480 \mathrm{~nm}$, and has approximately the same width as the emission spectrum in Figure 4.

In addition, we checked powdered samples of lead hydroxide $\left(\mathrm{Pb}(\mathrm{OH})_{2}\right)$ for scintillation, and found that its scintillation light output is less than $1 \%$ of basic lead carbonate [5]. Since basic lead carbonate differs from pure $\mathrm{PbCO}_{3}$ only by the addition of lead hydroxide and the scintillation properties of cerussite are very similar to those of basic lead carbonate, we therefore conclude that the $\mathrm{PbCO}_{3}$ molecule is the active scintillator in both cerussite and basic lead carbonate and that the inclusion of lead hydroxide does not affect its scintillation properties. This also implies that the scintillation seen in the cerussite crystals is not caused by impurities in the crystal.

\section{Synthetic Crystal Growth}

As mentioned earlier, all measurements were made with crystals cut from a single naturally formed sample of $\mathrm{PbCO}_{3}$, pictured in Figure 7. This sample has many black inclusions and internal cracks, which reduce the clarity of the crystal and lower its light collection efficiency. Therefore, the light output measurement is likely to be affected by the relatively poor optical quality of the cerussite crystal and the other measurements presented in this paper will probably not be affected. We hope that the light output presented here is a lower limit, and that the light output

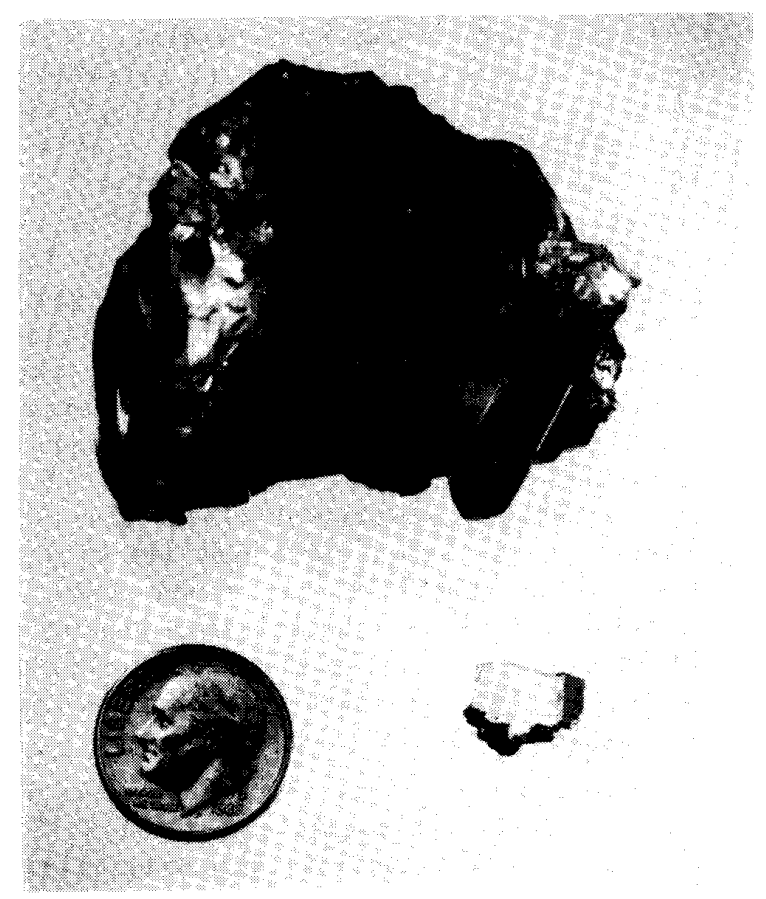

Figure 7: Photograph of the Cerussite Crystal

CBB 897-6098

of this material will increase once it is learned how to grow optical quality single crystals of pure $\mathrm{PbCO}_{3}$.

Growing pure synthetic crystals is complicated because the decomposition temperature of $\mathrm{PbCO}_{3}$ is lower than its melting point. However, it may be possible to grow optical quality crystals using a hydrothermal method, similar to the process used to grow single quartz crystals. Lead carbonate is slightly soluable in hot water, with its solubility increasing with increasing temperature. Therefore $\mathrm{PbCO}_{3}$ is dissolved in $300^{\circ} \mathrm{C}$ water at approximately 1000 atmospheres pressure, and a $50^{\circ} \mathrm{C}$ temperature gradient is placed across this solution, forming $\mathrm{PbCO}_{3}$ crystals at the colder end of the container [6].

It may also be possible to grow $\mathrm{PbCO}_{3}$ crystals from the melt by driving the reverse of decomposition reaction. $\mathrm{PbCO}_{3}$ breaks down into $\mathrm{PbO}$ and $\mathrm{CO}_{2}$ when it is heated, so if it were heated under several thousand atmospheres of $\mathrm{CO}_{2}$ pressure, the rate of the reverse of the decomposition reaction may increase enough to equal or exceed the decomposition rate. If this can be achieved, optical quality crystals can be grown using more conventional zonerefinement or Czochralski crystal growth techniques. This method has been applied with some success to grow optical quality $\mathrm{CaCO}_{3}$ crystals [7].

\section{Conclusions}

Lead carbonate is a newly discovered, heavy, inorganic scintillator. Its density of $6.6 \mathrm{~g} / \mathrm{cm}^{3}$ is similar to that of 
BGO $\left(7.1 \mathrm{~g} / \mathrm{cm}^{3}\right)$, and the attenuation length for $511 \mathrm{keV}$ photons is the same as BGO $(1.1 \mathrm{~cm})$. Its fastest decay component $(5.6 \pm 1 \mathrm{~ns})$ is slower than the $0.8 \mathrm{~ns}$ "fast" component of $\mathrm{BaF}_{2}$, but considerably faster than $\mathrm{BGO}$ ( $300 \mathrm{~ns}$ ) or the "slow" component of $\mathrm{BaF}_{2}(620 \mathrm{~ns})$. The $\mathrm{PbCO}_{3}$ emission spectrum peak is centered at $475 \mathrm{~nm}$, and so its emissions can be detected with good efficiency both by borosilicate glass photomultiplier tubes and PIN photodiodes. The scintillation light output is fairly low, approximately $9 \%$ of $\mathrm{BGO}$ at room temperature but increasing to twice that of $\mathrm{BGO}$ at $-40^{\circ} \mathrm{C}$. The light output measurements presented here were made with poor optical quality natural crystals, so we hope that the light output will be greater in synthetically grown pure $\mathrm{PbCO}_{3}$ crystals. The scintillation properties are not appreciably affected when $\mathrm{Pb}(\mathrm{OH})_{2}$ is added to form basic lead carbonate. It is unlikely that the scintillation is due to a trace impurity in this natural crystal, as similar scintillation properties are observed when a powdered sample of pure basic lead carbonate is excited with $22-\mathrm{keV}$ X-rays.

The combination of high density, short fluorescence lifetime, and reasonable light output suggest that $\mathrm{PbCO}_{3}$ would be useful for applications where high counting rates, good stopping power, and nanosecond timing are important, such as medical imaging and nuclear science. The absence of a significant "slow" fluorescent decay component implies that $\mathrm{PbCO}_{3}$ would be well suited for applications where counting rates as high as $10 \mathrm{Mhz}$ are expected.

\section{Acknowledgments}

We would like to thank Dr. Rupert Perera of Lawrence Berkeley Laboratory for many useful discussions, Mr. John Cahoon of Lawrence Berkeley Laboratory for invaluable technical support, and Dr. Charles Bouldin of the National Bureau of Standards for the use of beam line X23-A2 at the National Synchrotron Light Source. This work was supported in part by the Director, Office of Energy Research, Office of Health and Environmental Research of the U.S. Department of Energy, under contract No. DEAC03-76SF00098, and in part by Public Health Service Grant Numbers P01 HL25840 and R01 CA38086 awarded by the National Heart Lung and Blood and National Cancer Institutes, Department of Health and Human Services. Research carried out in part at the National Synchrotron Light Source, Brookhaven National Laboratory, which is supported by the U.S. Department of Energy, Division of Materials Sciences and Division of Chemical Sciences under contract No. DE-AC02-76CH00016.

Reference to a company or product name does not imply approval or recommendation of the product by the University of California or the U.S. Department of Energy to the exclusion of others that may be suitable.

\section{References}

[1] Robert C. Weast, editor. Handbook of Chemistry and Physics, page B108. The CRC Press, Inc., Boca Raton, FL, 1988.

[2] L.M. Bollinger and G.E. Thomas. Measurement of the time dependence of scintillation intensity by a delayedcoincidence method. Rev. Sci. Instr. 32, 1044-1050 (1961).

[3] M. Moszyński and B. Bengtson. Light pulse shapes from plastic scintillators. Nucl. Instr. and Meth. 142, 417-434 (1977).

[4] I. Holl, E. Lorenz, and G. Mageras. A measurement of the light yield of common inorganic scintillators. IEEE Trans. Nucl. Sci. NS-35, 105-109 (1988).

[5] S.E. Derenzo, W.W. Moses, et al. Prospects for new inorganic scintillators. IEEE Trans. Nucl. Sci. NS-37, (1990). (To be published in these proceedings).

[6] D.F. Croxall, R. Lambert, and R.C. Kell. United Kingdom Patent 1,468,052. 1977.

[7] E.H. Baker, F.J. Nazareth, and D.S. Robertson. Growth of crystalline calcite from a $\mathrm{CaCO}_{3}-\mathrm{CaO}$ melt. J. Crystal Growth 71, 197-202 (1985). 